\title{
Modeling and optimization of production and distribution of drinking water at VMW
}

Derek Verleye and El-Houssaine Aghezzaf

\begin{abstract}
We develop and discuss an operational planning model aiming at minimizing production and distribution costs in large drinking water networks containing buffers with free inflow. Modeling drinking water networks is very challenging due of the presence of complex hydraulic constraints, such as friction losses and pump curves. Non-linear, non-convex constraints result from the relationships between pressure and flow in power terms. Also, binary variables are needed to model the possibility of free inflow or re-injection of water at reservoirs. The resulting model is thus a non-convex Mixed-Integer Non-Linear Program (MINLP). A discrete-time setting is proposed to solve the problem over a finite horizon made of several intervals. A commercial solver, BONMIN, suited for convex MINLP models is used to heuristically solve the problem. We are able to find a good solution for a small part of an existing network operated by the Vlaamse Maatschappij voor Watervoorziening (VMW), a major drinking water company in Flanders.
\end{abstract}

\section{Introduction}

After an optimal design of the drinking water network of water production and distribution systems, further economic efficiency can be reached through optimal management of operational activities such as production scheduling and pump switching. Most drinking water companies neglect these savings by operating their networks based upon experience. In this regard, an operational support model that minimizes production and distribution costs over a finite horizon is extremely use-

D. Verleye $\cdot$ E.-H. Aghezzaf

Department of Industrial Management, Faculty of Engineering, Ghent University,

Building 903, Technologiepark, 9052 Zwijnaarde

e-mail: derek.verleye@ugent.be

E.-H. Aghezzaf

e-mail: elhoussaine.aghezzaf@ugent.be 
ful. Distribution and production in drinking water networks can be modeled as a minimum cost flow problem (Ahuja et al, 1993; Bertsekas, 1998) with many side constraints. The arcs represent pipelines, whereas nodes are used to represent junctions, buffers etc. Conservation of flow and bounds on the flow rate are imposed as restrictions. This model is expanded with more complicated constraints resulting from the network hydraulics.

Early research papers on this subject handle energy-efficient operation of pumps (Pezeshk et al, 1994; Ulanicki et al, 1993), possibly in combination with guaranteed supply by optimal management of water inventory in buffers (Crawley and Dandy, 1993). Nonlinearity of the objective function is an immediate consequence of the 'power term', which is a function of two main variables of the system: pump head and flow rate. In D'Ambrosio (2009), an approach to linearize this term is proposed. The resulting MILP for a hydro scheduling commitment was hereby successfully solved. The optimization of large-scale drinking water network with multiple production centers is a less studied topic (Burgschweiger et al, 2009, 2008). The authors present a complete model for the drinking water supply in Berlin, using sophisticated functions to describe the friction losses in pipes as well as accurate mathematical models for pump curves. Their formulation results in a MINLP model, which is very difficult to solve for such a large network. Hence, the model is approximated by a practical useful NLP (nonlinear problem) formulation. These authors state however, that more complicated MINLP models will become practical in the future with improvements in computing technology and mathematical algorithms.

In Verleye (2010), the first steps in developing such a model are taken. This paper summarizes the key aspects of this work. A specific component is free inflow and reinjection of water at reservoirs, requiring additional binary variables in the model. The relationship between flow and head in pressure losses and the power term lead to nonlinear, non-convex constraints. The resulting model is tested on a small part of the existing network of VMW (Vlaamse Maatschappij voor Watervoorziening), the largest drinking water company in Flanders. The commercial solver BONMIN (Bonami and Lee, 2007) is used to generate a suboptimal solution over a horizon of one day, divided in several discrete periods, in a reasonable computation time.

\section{Description of the model}

In this section, the structure as well as the different components in the supply network are defined. The different hydraulic constraints will be mathematically formulated for each of these components. Note that we only consider a reduced supply network in the province West Flanders (Flanders, Belgium) which is showed in Fig. 1. The supply network is the pipeline structure wherein large volumes of fresh water are sent from the production station to the buffers, delivery points and junctions with the distribution network. The latter one consist of the pipes with small diameters through which water is distributed to the end user. In this report, demand in the distribution network is clustered in so called demand parameters in the supply 
network. The supply network is modeled as a graph $G=[K, L]$, where $K$ denotes the set of nodes and the set $L$ contains all the pipes in the network. Nodes will be indicated by the superscript $i$, whereas pipes connecting nodes $i$ and $j$ are indicated by superscript $i j$.

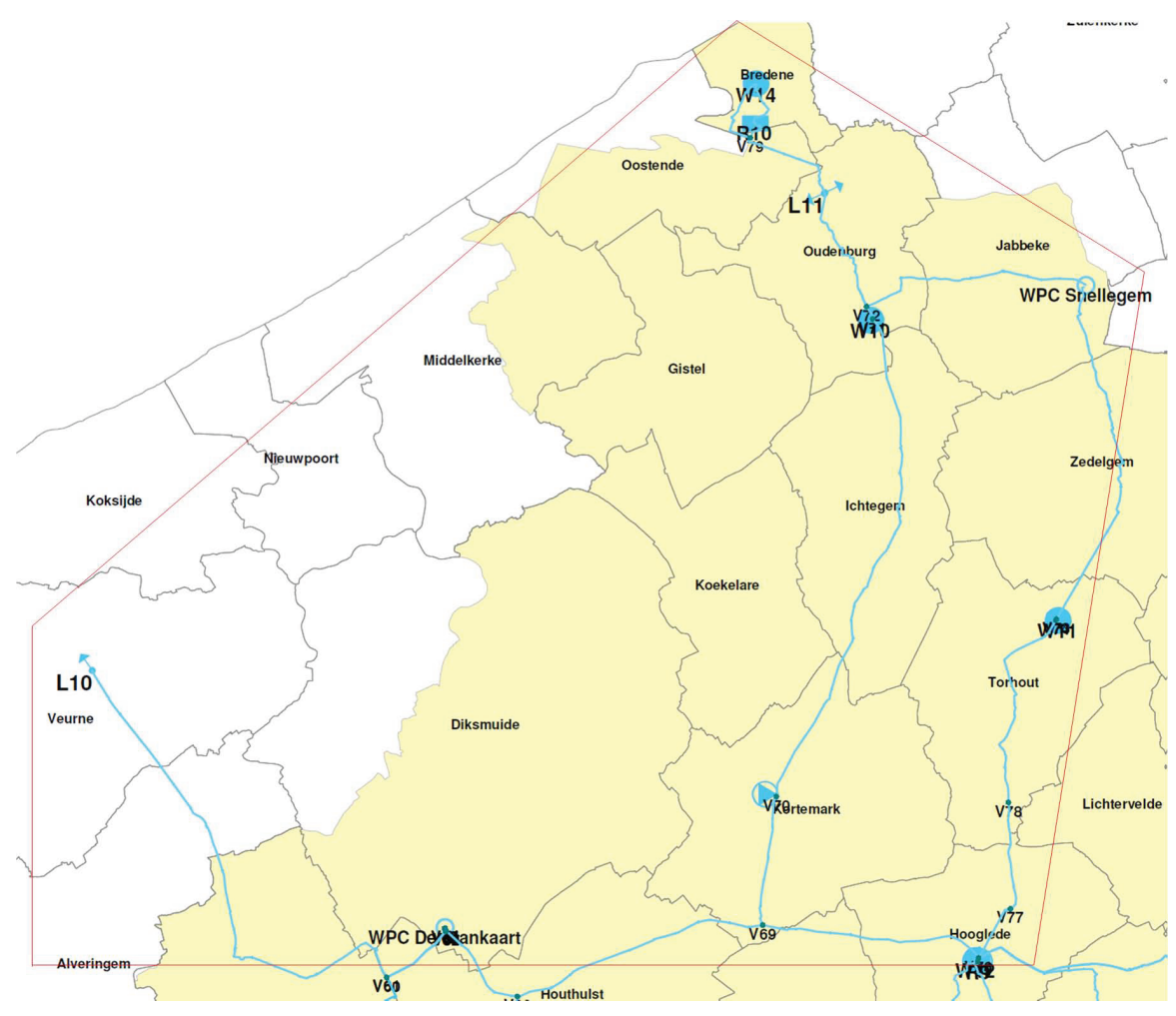

Fig. 1 Test configuration with junctions (V), reservoirs (R), water towers (W) and production centers (WPC)

In what follows, the time division in discrete periods is explained. An overview of the most important symbols is given. Next, the constraints in different components in the considered network during each of these periods, are described. Afterwards, the goal function is defined.

\subsection{Discrete time setting}

As an optimization over a period of at least one day is desired, a day is divided into discrete periods during each of which the state of the network is assumed to 
be constant. Because a calculation of the network state at every hour is very timeconsuming, a division of a day as in Fig. 2 is proposed. This division is based upon a typical hourly demand pattern and the different day and night tariffs for electricity. We will denote an interval by $t \in[1, T]$, where $T$ represents the number of periods. The length of each period is denoted by $\tau_{t}$.

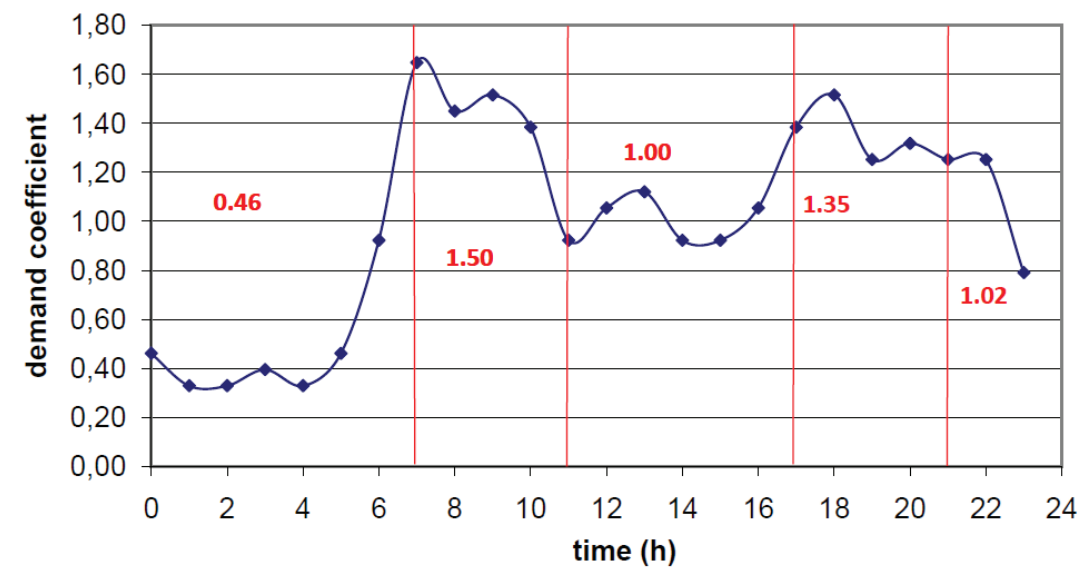

Fig. 2 Discrete time intervals based upon demand coefficients and electricity tariffs

\subsection{System components and restrictions}

Throughout this section restrictions will be written per period, for each $t \in 1$...T Furthermore, the following notation will be used:

- $K_{B}$ : set of buffers

- $L_{G}$ : set of pipes wherein water flows gravitationally out of a buffer

- $L_{L}$ : set of pipes wherein no pump is active

- $L_{R}$ : set of pipes wherein a raw water pump is active

- $L_{P}$ : set of pipes wherein a pure water pump is active

- $H$ : the piezometric head as the sum of the geometric height $h$ and the manometric water pressure $p / \gamma(\mathrm{m})$

- $Q$ : flow through a pipe $\left(\mathrm{m}^{3} / \mathrm{h}\right)$

- $B$ : fluid level in tank at the end of a period (m)

- $V$ : volume in tank at the end of a period $\left(\mathrm{m}^{3}\right)$

- $M$ : mean head of water in tank (m)

- $\Delta H$ : pump head (m)

- $P$ : power delivered by pump $(\mathrm{W})$ 
The main decision variables are $Q_{i j}, i j \in L_{R}$, the amount of water produced in the water production centers and $\Delta H$, the head delivered by each pump. Knowledge of the value of these variables will allow operators to optimally control the network. All other variables are thus dependent on these two decision variables.

\subsubsection{Node modeling}

We distinguish following nodes:

1. Junctions and delivery points

2. Buffers

3. Basins

Junctions and delivery points

In this paper, a junction is defined as the place where the material or diameter of a pipe changes, where the pipe splits up in two or more sections or where a nonzero demand parameter $v$ (in $\mathrm{m}^{3} / \mathrm{h}$ ) is assigned to a point in the network.

Apart from conservation of mass, limits to the allowed pressure are also imposed. As a general rule, no negative pressures are allowed and the manometric pressure cannot exceed 10 bar. As these limits are calculated in the junctions, we assume that the height of the actual pipeline is in between that of the endpoints. Otherwise stated, the pipelines are monotone ascending or descending in between two junctions such that the pressure limits are respected everywhere.

Delivery points are situated at the border of two different drinking water networks. If one of the parties has difficulties of delivering water without capacity expansion or the use of boosters and the other party is able to easily deliver extra water in this point, a long-term contract can be signed. A delivery parameter, that can be either positive or negative, is assigned to these nodes.

\section{Buffers}

Buffers play a major role in drinking water networks. Apart from their storage capacity, they are of a major economical importance. At night, when the energy tariff is low, water can be stored in the reservoir using pumps. The next day this water can flow gravitationally back into the network to meet part of the demand. In this way, high energy costs due to excessive pumping during the day are avoided.

In buffers, we distinguish both pure water tanks and water towers. The latter one can be interpreted as an elevated tank on an underneath construction, thus falling under the same category.

The most general representation of a buffer is given in Fig. 3. Despite its complexity, it is modeled as a single conceptual node. At the entrance (connection (a)) water flows in or out at a variable head. At the exit (connection (c)) water is pumped 
out at the pressure exercised by the fluid level of the tank. Water storage and distribution take place at the tank itself.

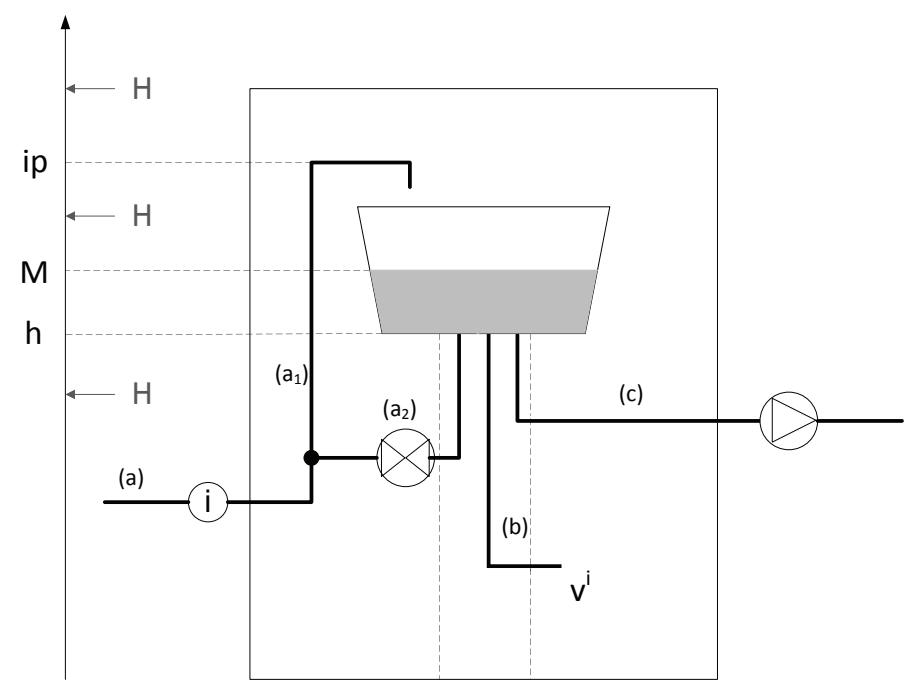

Fig. 3 Model of a buffer. Depending on the variable value of the head $(H)$ at the entrance $i$, water can flow either in or out.

The conservation of mass is expressed as:

$$
\sum_{k:(k, i) \in L} Q_{t}^{k i}-\sum_{j:(i, j) \in L_{L} \cup L_{R}} Q_{t}^{i j}=I_{t}^{i+}-I_{t}^{i-}
$$

Here, $I^{i+}$ and $I^{i-}$ represent in- and outflow at the buffer, respectively. The necessity of these two additional variables will soon be explained.

Other relationships that take place at the buffer will be explained using Fig. 3. At (a), water in the connected pipes will flow either in or out of the buffer, depending on the head $H$. Since water can flow freely into the tank, actual inflow only takes place through connection (a1) in periods where the head is bigger than the height of the inflow point $i p$. Under some circumstances, the mean head of the water $M$ in the tank is higher than the head at the entrance $i$. As a consequence, water will now be re-injected into the pipe network through connection (a2). This is expressed by the following relations:

$$
\begin{aligned}
& H_{t}^{i} \geq i p^{i} \Rightarrow I_{t}^{i+} \geq 0 \\
& H_{t}^{i} \leq M_{t}^{i} \Rightarrow I_{t}^{i-} \geq 0
\end{aligned}
$$


By introducing some binary variables, we translate these relations into constraints.

If the head at the entrance of the buffer is in between these values, neither in- nor outflow will occur. In this case, the operator is nevertheless able to open the bypass at (a2) in order to allow water to flow into the tank. Doing this has some unwanted qualitative effects and forces us to add extra binary variables, so we decide not to include this bypass in our model.

At (c), boosters or fresh water pumps transporting water out of the buffer as well as pipes in which water flows out gravitationally, induce an outflow $U^{i-}$ :

$$
\sum_{j:(i, j) \in L_{P} \cup L_{G}} Q_{t}^{i j}=U_{t}^{i-}
$$

An important variable is the tank volume at the end of a period, $V^{i}$, as it links two subsequent periods through the filling rate:

$$
V_{t}^{i}=V_{t-1}^{i}+\left(I_{t}^{i+}-I_{t}^{i-}-U_{t}^{i-}-v_{t}^{i}\right) \tau_{t}
$$

Notice that a buffer may directly be feeding the distribution network with demand $v^{i}$ through (b).

Necessary initial and terminal conditions are:

$$
\begin{gathered}
V_{0}^{i}=V^{i}(0) \\
V_{T}^{i} \geq V^{i}(0)
\end{gathered}
$$

where $V^{i}(0)$ is the tank volume present at the start of the simulation. The second constraint prevents the optimal configuration from depleting all buffers in the last period.

The fluid level in the tank at the end of period $t$ is defined as

$$
B_{t}^{i}=\frac{V_{t}^{i}}{A^{i}}
$$

The cross section of the tank, $A^{i}$, is approximated as a constant circle over the entire height, despite the fact that the tank is possibly conic. The fluid level is restricted by a lower bound due to quality considerations and an upper bound equal to the total height of the tank.

To obtain an approximation of the mean head of the water in the tank during a period, the mean fluid level is augmented with the geometric height of the tank floor:

$$
M_{t}^{i}=h^{i}+\frac{B_{t}^{i}+B_{t-1}^{i}}{2}
$$


Basins

In basins, raw water is captured at surface water treatments. In an optimization over one period, they act as an infinite source of water. Therefore, no restrictions take place at this node. By subtracting the outflow, the volume at the end of the optimization is generated in the output in order to know how much reserve is left.

\subsubsection{Pipe modeling}

Friction losses in pipes are calculated by the formula of Darcy-Weisbach for turbulent flow:

$$
\Delta p=\lambda \frac{l}{d} \rho \frac{v^{2}}{2}
$$

where $l$ denotes the length of the pipe and $d$ is the inner diameter. The (dimensionless) friction coefficient $\lambda$ depends on the value of the Reynolds number. Here, we work with the (simplified) law of Prandtl-Kármán for hydraulically rough pipes:

$$
\lambda=\left(2 \log \frac{k}{3.71 d}\right)^{-2}
$$

where $k(\mathrm{~mm})$ represents the roughness of the pipe and is dependent of the material. Substituting $v=\frac{Q}{A}, A=\frac{\pi d^{2}}{4}$, the friction loss equation leads to:

$$
H_{t}^{i}-H_{t}^{j}=\frac{8 l^{i j}}{3600^{2} g \pi^{2}\left(d^{i j}\right)^{5}} \lambda^{i j} Q_{t}^{i j}\left|Q_{t}^{i j}\right|, \quad \forall(i, j) \in L_{L}
$$

for general pipes, and to

$$
M_{t}^{i}-H_{t}^{j}=\frac{8 l^{i j}}{3600^{2} g \pi^{2}\left(d^{i j}\right)^{5}} \lambda^{i j}\left(Q_{t}^{i j}\right)^{2}, \forall(i, j) \in L_{G}
$$

for water flowing gravitationally out of a buffer.

The formulas (8) and (9) will lead to an underestimation of the friction losses for small values of the flow (up to $20 \mathrm{~m}^{3} / \mathrm{h}$ ). In the supply network, such small values for the flow are not common. This justifies the approximation. For a more precise calculation of the friction losses, see Burgschweiger et al., 2000.

The pressure losses for a specific pipe, calculated according to (8), are displayed in Fig. 4. This constraint is clearly non-convex.

A pipe that is out of use, must be excluded from the model. Hereto we add a binary control parameter $x$ for every pipe in the network. 


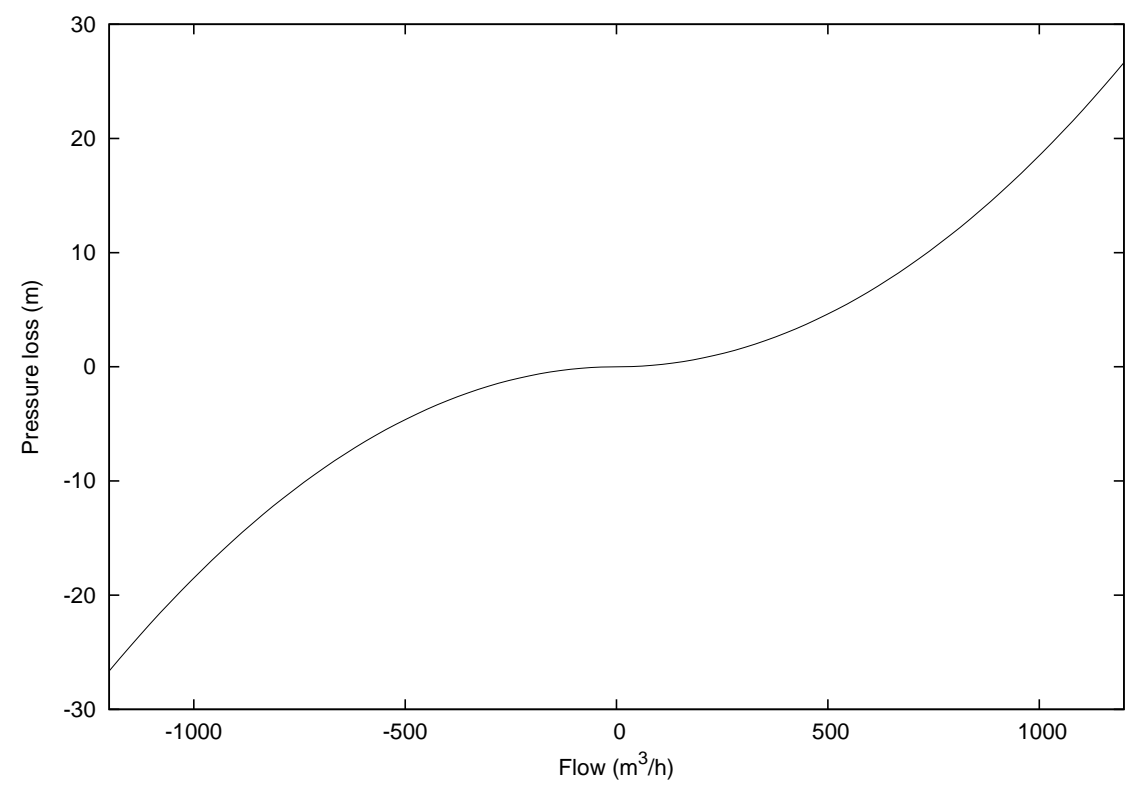

Fig. 4 Pressure losses in a pipe with $1=5 \mathrm{~km}, \mathrm{~d}=500 \mathrm{~mm}, \mathrm{k}=0,5$

\subsubsection{Pump modeling}

We distinguish:

1. delivery pumps and boosters

2. raw water pumps

\section{Delivery pumps and boosters}

Delivery pumps add pressure to distribute the water from the fresh water basements at the production centers throughout the network. Boosters are placed on well chosen places in the network where extra pressure is needed due to friction losses. In the pipes where the pump is active, we find the following equations:

$$
\begin{gathered}
H_{t}^{i}-H_{t}^{j}=\frac{8 l^{i j}}{3600^{2} g \pi^{2}\left(d^{i j}\right)^{5}} \lambda^{i j}\left(Q_{t}^{i j}\right)^{2}-\Delta H_{t}^{i j}, \forall(i, j) \in L_{P}: i \in K \backslash K_{B} \\
M_{t}^{i}-H_{t}^{j}=\frac{8 l^{i j}}{3600^{2} g \pi^{2}\left(d^{i j}\right)^{5}} \lambda^{i j}\left(Q_{t}^{i j}\right)^{2}-\Delta H_{t}^{i j}, \forall(i, j) \in L_{P}: i \in K_{B}
\end{gathered}
$$

where $\Delta H^{i j}$ is the head added by the pump. This head depends on the flow that goes through the pump and can be derived from the pump curve. The operating 
point is the point where this curve and the system curve - the pressure loss curve augmented with the static hight difference - meet. Figure 5 shows this principle for a delivery pump which is currently in use by VMW in West Flanders.

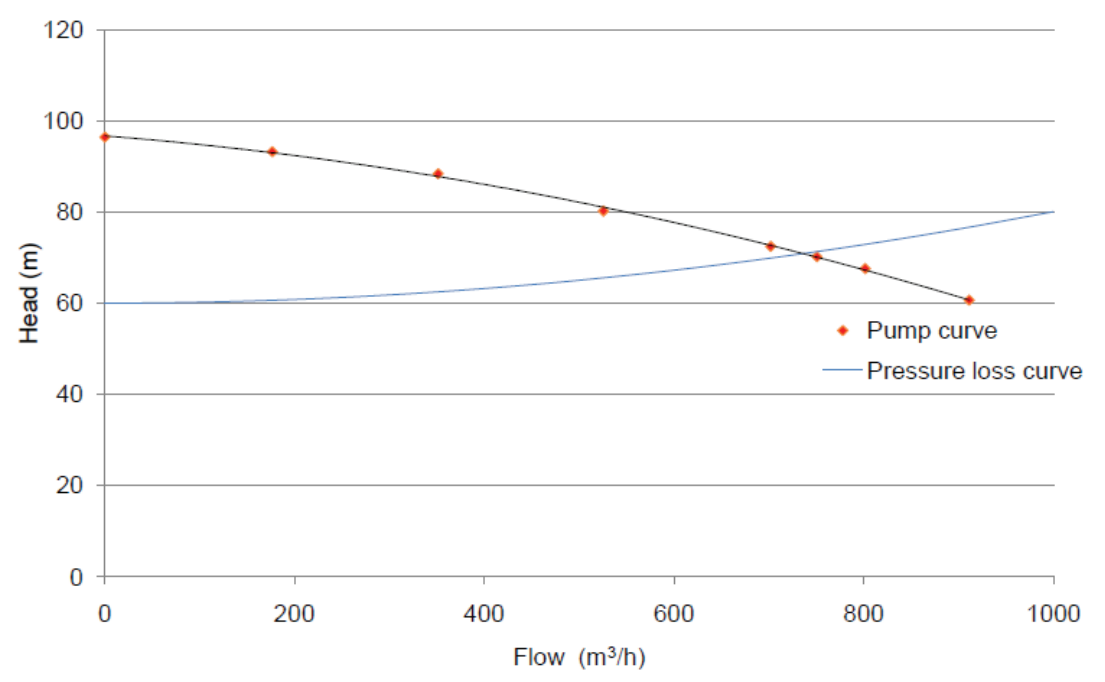

Fig. 5 Operating point of a pump at a given pressure loss and static height difference

To correctly model this curve, an extensive study is needed. However, such a study does not fit in the scope of this research. Since most of the pumps in the network are variable speed pumps, we undertake a different approach. The working region of a variable speed pump is much larger. Moreover, the flow is within a wide range of values for a fixed value of the pressure, as can be seen from Fig. 6. That is why we force the outlet pressure of this pumps to be fixed at a value $u^{i j}$, giving:

$$
\begin{array}{r}
\Delta H_{t}^{i j}=x^{i j}\left(u^{i j}-H_{t}^{i}\right), \quad \forall i \in K \backslash K_{B} \\
\Delta H_{t}^{i j}=x^{i j}\left(u^{i j}-M_{t}^{i}\right), \quad \forall i \in K_{B}
\end{array}
$$

$x$ is, again, a binary control parameter that takes the value 0 if the pump is not working. The values for $u$ are divided in a winter and a summer regime and are chosen with the aid of the experienced operators at VMW.

For pumps with fixed speed, we opt for a linear approximation of the pump curve. Departing from three fixed points on the pump curve, $[q(1), \Delta h(1)],[q(2), \Delta h(2)]$, $[q(3), \Delta h(3)]$, with $q(1)<q(2)<q(3)$ and $h(1)>h(2)>h(3)$, we note:

$$
\begin{array}{r}
U_{t}^{i j}(1) \leq W_{t}^{i j}(1), U_{t}^{i j}(2) \leq W_{t}^{i j}(1)+W_{t}^{i j}(2), U_{t}^{i j}(3) \leq W_{t}^{i j}(2) \\
U_{t}^{i j}(1)+U_{t}^{i j}(2)+U_{t}^{i j}(3)+W_{t}^{i j}(3)=1 \\
W_{t}^{i j}(1)+W_{t}^{i j}(2)=1
\end{array}
$$




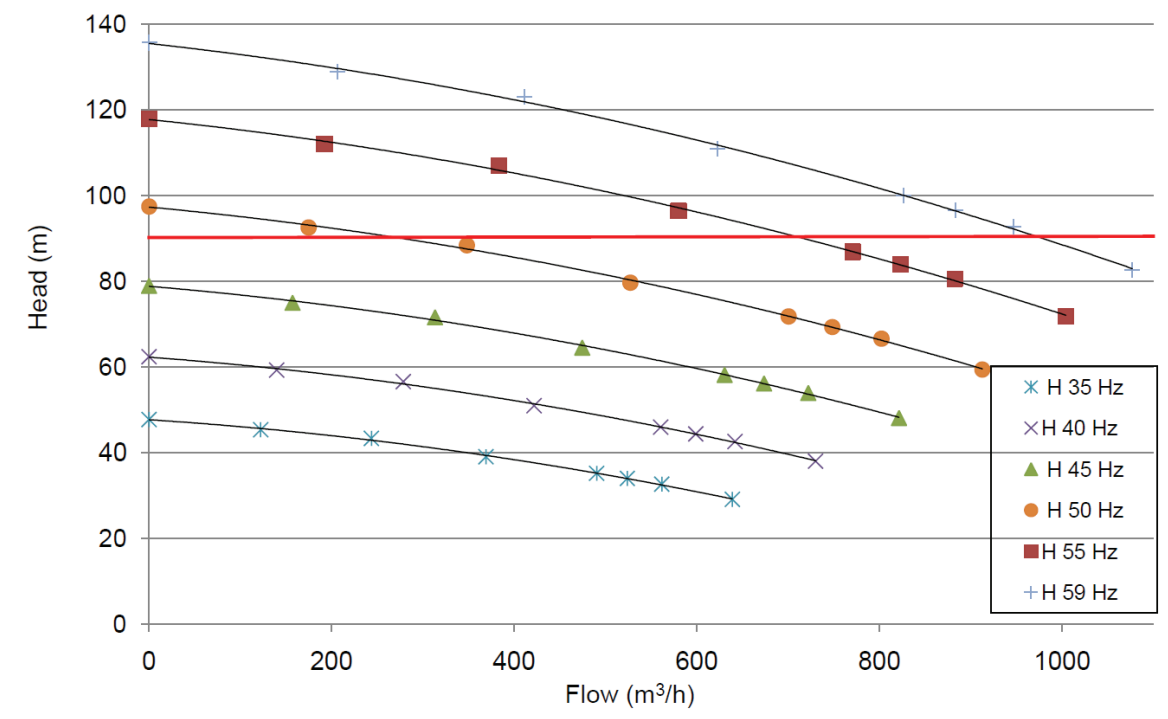

Fig. 6 Pump curve at different frequencies, fixed pressure of $90 \mathrm{~m}$

$$
\begin{array}{r}
Q_{t}^{i j}=x^{i j}\left[U_{t}^{i j}(1) q^{i j}(1)+U_{t}^{i j}(2) q^{i j}(2)+U_{t}^{i j}(3) q^{i j}(3)\right] \\
\Delta H_{t}^{i j}=x^{i j}\left[U_{t}^{i j}(1) \Delta h^{i j}(1)+U_{t}^{i j}(2) \Delta h^{i j}(2)+U_{t}^{i j}(3) \Delta h^{i j}(3)\right]
\end{array}
$$

In this formulation, the variables $U$ are real, whereas $W$ are binary. $W(3)$ is needed to allow the solution $Q=\Delta H=0$.

The power is given by

$$
P_{t}^{i j}=\frac{2,73 \Delta H_{t}^{i j} Q_{t}^{i j}}{\eta_{t}^{i j}}
$$

for all pure water pumps. Here, $\eta$ denotes pump efficiency and is dependent on the pump's operating point. For simplicity we state $\eta_{t}^{i j}=\eta^{i j}=0,65$ which is a rather safe assumption.

Finally, the flow through the pump has to lie within the allowable range:

$$
x^{i j} Q^{i j}(\min ) \leq Q_{t}^{i j} \leq x^{i j} Q^{i j}(\max )
$$

\section{Raw water pumps}

Those pumps which push water from the basins all the way through the treatment at the production center are not explicitly modeled. The reason hereto is the fact that the total pressure needed through to get water through these treatment steps is almost constant. We thus consider the cost of the energy delivered by these pumps 
as part of the production cost and thus linearly dependent with the production flow. The only restrictions are the treatment capacities and raw water contracts as an upper bound to the total produced fresh water.

\subsection{Goal function}

As stated before, the goal function consists of two terms: a production and an energy cost. The production cost, $k p$ (in $€ / \mathrm{m}^{3}$ ), is time-independent and consists of the costs of chemicals, taxes and the electricity cost. Not only the energy for lighting and treatment is considered, but also for operating the raw water pumps at a fixed pressure. The energy cost of the other pumps is calculated with ke, the timedependent energy tariff (in $€ / \mathrm{kWh}$ ). The total cost function that is to be minimized can thus be written as:

$$
\operatorname{Min} \sum_{t=1}^{T}\left(\sum_{(i, j) \in L_{P}} P_{t}^{i j} \frac{k e_{t}}{1000}+\sum_{(i, j) \in L_{R}} Q_{t}^{i j} k p^{i j}\right) \tau_{t}
$$

\section{Testing and results}

The resulting MINLP model is non-convex due to the pressure loss constraint. Therefore, a global optimal solution is not guaranteed. As a programming language, $A M P L$ is used and the problem is solved using the open-source solver BONMIN. As this solver is suited for convex problems, one can expect a heuristic approach of the optimal solution.

Firstly, a configuration is made on which the model will be tested. An optimization is done over a horizon of two periods. Once the working of the model is validated, the existing network in West Flanders is optimized. An optimization is executed over a period of 24 hours. While optimizing over 3 periods does not pose any difficulties, no solution is found from 4 periods onwards. The reason hereto are the fixed pressure restrictions at variable speed pumps. During periods of high demand, friction losses take on high values due to the large pumped flows. In some cases, the fixed pressure may be insufficient to compensate for these losses. Therefore, restrictions 12 and 13 are dropped. This will allow free combinations of flow-head values without considering the actual operating area of the pump.

The test configuration (see also Fig. 1) consists of 2 production centers, 20 junctions, 1 delivery point and 5 buffers, from which 4 are water towers and one is a reservoir. All these nodes are connected by 28 pipes, 2 delivery pumps and 2 boosters. In an acceptable calculation time, we are able to find an optimal solution for a relatively small network. On a HP Pavilion dv6700, $1.83 \mathrm{GHz}$ Processor with $3 \mathrm{~GB}$ RAM we found a solution within a time of $885 \mathrm{~s}$. Despite the 'free' behavior of the variable speed pumps, flow-head values were within decent ranges. Nevertheless, 
the pump curves should be mathematically formulated in a future stadium. Not only will this prevent solutions which are infeasible in practice, the bounds on these two main variables will certainly allow a faster convergence to a (sub)optimal solution.

Some important results can be derived from the optimal solution. All clean water tanks maintain a maximum fluid level during all periods, except the final one. This can be explained through the gain in energy efficiency from the smaller head that delivery pumps behind those reservoirs have to add. During periods of low energy costs, all water towers maintain a minimal fluid level. During periods of low energy cost, these towers are filled back again. These facts lead to the graph in Fig. 7, which displays the total pumping power together with the energy tariff.

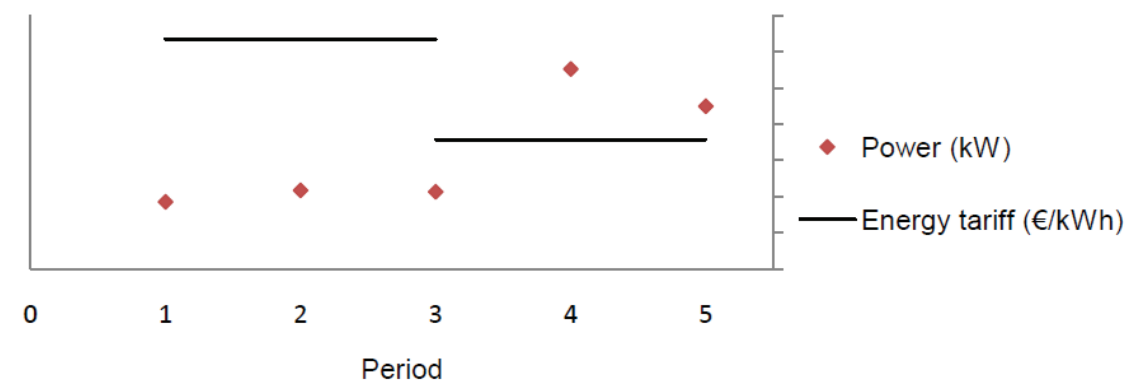

Fig. 7 Pump power versus energy tariff

\section{Conclusion}

A model for operative planning in a drinking water network over a finite horizon was constructed. An important component is the basin with free inflow and outflow possibilities, as it is the major link between subsequent periods. The result is a nonconvex MINLP model that is based on several simplifications, such as an empirical approximation of the friction coefficient. To solve the problem, we relied on the open-source solver BONMIN (Bonami and Lee, 2007), which was able to generate a solution for a part of the existing network within several minutes. The optimal configuration gives good results concerning the time-dependent electricity tariffs, mostly due to the efficient filling and depleting of the basins.

An important step for further improvement is a detailed modeling of the characteristic pump curves. At the same time, the pressure losses should be calculated with more precision. Additional components such as valves can be modeled as well. Once the model accurately describes the complete network hydraulics, attempts shall be undertaken to minimize the calculation time. This can be accomplished by smart 
reformulations of the complicating constraints. Additionally, the use of other exact and heuristic methods will be tested.

\section{References}

Ahuja RK, Magnanti TL, Orlin JB (1993) Network Flows: Theory, Algorithms, and Applications. Prentice Hall

Bertsekas DP (1998) Network Optimization: Continuous and Discrete Models. Optimization and Computation Series, Athena Scientific, Post Office Box 391 Belmont, Massachusetts

Bonami P, Lee J (2007) BONMIN Users‘ Manual

Burgschweiger J, Gnädig B, Steinbach MC (2008) Optimization models for operative planning in drinking water networks. Optim Eng 10:43-73

Burgschweiger J, Gnädig B, Steinbach MC (2009) Nonlinear programming techniques for operative planning in large drinking water networks. Open Appl Math J 3:14-28

Crawley PD, Dandy GC (1993) Optimal operation of multiple-reservoir system. J Water Resour Plan Manage 119(1):1-17

D’Ambrosio C (2009) Application-oriented mixed integer non-linear programming. Phd thesis, University of Bologna

Pezeshk S, Helweg OJ, Oliver KE (1994) Optimal operation of ground-water supply distribution systems. J Water Resour Plan Manage 120(5):573-586

Ulanicki B, Rance JP, Davis D, Chen S (1993) Computer-aided optimal pump selection for water distribution networks. J Water Resour Plan Manage 119(5):542562

Verleye D (2010) Modellering en optimalisatie van waterproductie en -verdeling bij de vlaamse maatschappij voor watervoorziening. Master thesis, University of Ghent 